Pacific Journal of Mathematics

STRONG RESULT FOR REAL ZEROS OF RANDOM

N. Misura, N. N. Maya and Swadeenananda Pattanaya 


\title{
STRONG RESULT FOR REAL ZEROS OF RANDOM POLYNOMIALS
}

\author{
M. N. Mishra, N. N. Nayak and S. Pattanayak
}

Let $N_{n}$ be the number of real zeros of $\sum_{r=0}^{n} a_{r} X_{r} x^{r}=0$ where $X_{r}$ 's are independent random variables identically distributed belonging to the domain of attraction of normal law; $a_{0}, a_{1}, a_{2} \cdots a_{n}$ are nonzero real numbers such that $\left(k_{n} / t_{n}\right)=o(\log n)$ where $k_{n}=\max _{0 \leqq r \leqq n}\left|a_{r}\right|$ and $t_{n}=\min _{0 \leqq r \leqq n}\left|a_{r}\right|$. Further we suppose that the coefficients have zero means and $P\left\{X_{r} \neq 0\right\}>0$. Then there exists a positive integer $n_{0}$ such that

$$
P\left\{\sup _{n>n_{0}}\left(N_{n} / D_{n}\right)<\mu\right\}>1-\mu^{\prime}\left\{\log \left(\left(k_{n_{0}} / t_{n_{0}}\right) \log \log n\right) / \log n_{0}\right\}^{1-\varepsilon / 2}
$$

for $n>n_{0}$ and $1>\varepsilon>0$ where $D_{n}=\left(\log n / \log \left(k_{n} / t_{n}\right) \log \log n\right)^{(1-\varepsilon) / 2}$.

1. Let $N_{n}$ be the number of real roots of a random algebraic equation

$$
\sum_{r=0}^{n} X_{r} x^{r}=0,
$$

where $X_{r}$ 's are independent, identically distributed random variables. The problem of finding the lower bound of $N_{n}$ has been considered by various authors. Considering the coefficients as normally distributed or uniformly distributed in $[-1,1]$, assuming the values +1 or -1 with equal probability Littlehood and Offord [8] have shown that $N_{n}>\mu \log n / \log \log n$ except for a set of measure at most $\mu^{\prime} / \log n, n$ being sufficiently large. Evans [4] has studied the strong version of Littlehood and Offord and has shown that in case of Gaussian distributed coefficients $N_{n}$ is greater than $\mu \log n / \log \log n$ except for a set of measure at most $\mu^{\prime}\left(\log \log n_{0} / \log n_{0}\right)$ for $n>n_{0}$. The above result is strong in the following sense.

Theorem of Littlehood and Offord is of the form

$$
P\left\{\left(N_{n} / D_{n}^{\prime}\right)<\mu\right\} \longrightarrow 1 \text { as } n \longrightarrow \infty,
$$

where $D_{n}^{\prime}=\log n / \log \log n$. But the theorem of Evans is of the form

$$
P\left\{\sup _{n>n_{0}}\left(N_{n} / D_{n}^{\prime}\right)<\mu\right\} \longrightarrow 1 \text { as } n_{0} \longrightarrow \infty .
$$

Considering the coefficients of $\sum_{r=0}^{n} a_{r} X_{r} x^{r}=0$ as symmetric stable variables Samal and Mishra [13] have shown that 


$$
P\left\{\left(N_{n} / D_{n}^{*}\right)<\mu\right\}>1-\frac{\mu^{\prime}}{\left\{\log \left(k_{n} / t_{n}\right) \log n\right\}(\log n)^{\alpha-1}} \text { if } 1 \leqq \alpha<2
$$

and

$$
>1-\frac{\mu^{\prime} \log \left(k_{n} / t_{n}\right) \log n}{\log n} \text { if } \alpha=2,
$$

where $k_{n}=\max _{0 \leqq r \leqq n}\left|a_{r}\right|, t_{n}=\min _{0 \leqq r \leqq n}\left|a_{r}\right|$ and $D_{n}^{*}=\left(\log n / \log \left(\left(k_{n} \mid\right.\right.\right.$ $\left.\left.\left.t_{n}\right) \log n\right)\right)$. Samal and Mishra [13] have studied the strong version of the above theorem and have shown that $P\left\{\sup _{n>n_{0}}\left(N_{n} / D_{n}^{*}\right)<\mu\right\}$

$$
>1-\frac{\mu}{\left\{\log \left(\log n_{0} / \log \left(k_{n_{0}} / t_{n_{0}}\right) \log n_{0}\right)\right\}^{\alpha-1}} \text { where } \alpha>1 .
$$

Mishra and Nayak [9] have proved that

$$
P\left\{\left(N_{n} / D_{n}^{*}\right)<\mu\right\}>1-\frac{\mu^{\prime}}{\left\{\log \left(\left(k_{n} / t_{n}\right) \log n\right)\right\}(\log n)^{1-\varepsilon}}
$$

for every positive $\varepsilon<1$, when the coefficients belong to the domain of attraction of the normal law.

Object of this paper is to show that

$$
\begin{aligned}
P\left\{\sup _{n>n_{0}}\left(N_{n} / D_{n}\right)\right. & <\mu\} \\
& >1-\mu^{\prime}\left\{\frac{\log \left(\left(k_{n_{0}} / t_{n_{0}}\right) \log \log n_{0}\right)}{\log n_{0}}\right\}^{1 / 2}
\end{aligned}
$$

for $0<\varepsilon<1$, when the coefficients belong to the domain of attraction of the normal law. Therefore it is a strong result of Mishra and Nayak.

Throughout this paper we shall denote $\mu$ 's for positive constants which may assume different values in different occurences and $V(\cdot)$ for the variance of a random variable.

2. In the sequel we shall need the following definition, and theorem due to Karamata, (cf. Ibragimov and Linnik [6] p. 394), for the proof of our main result.

Definition. A function $H: \boldsymbol{R}_{+} \rightarrow \boldsymbol{R}_{+}$is called a slowly varying function if

$$
\lim _{x \rightarrow \infty} \frac{H(\gamma x)}{H(x)}=1,(\gamma>0) .
$$

We have a few characterization of the slowly varying functions due to Karamata.

By writting $H(1 / t)=h(t)$, we may define a slowly varying func- 
tion $h: \boldsymbol{R}_{+} \rightarrow \boldsymbol{R}_{+}$with the property that

$$
\lim _{x \rightarrow 0} \frac{h(\gamma x)}{h(x)}=1,(\gamma>0) .
$$

With this the Karamata theorem, (cf. Ibragimov and Linnik [6], p. 394), may be stated as follows.

THEOREM 1. A slowly varying function $h$ with the property (2.2) which is integrable on any finite interval may be represented in the form

$$
h(x)=c(x) \exp \left(-\int_{a}^{x} \frac{\bar{\varepsilon}(u)}{u} d u\right)
$$

where

$$
\lim _{x \rightarrow 0} c(x)=c \neq 0, \quad \lim _{x \rightarrow 0} \bar{\varepsilon}(x)=0 \text { and } a>0 .
$$

We establish the following formulae which will be necessary for the proof of the main theorem.

Let a sequence of independent and identically distributed random variables $\left\{X_{r}\right\}$ with mean zero belong to the domain of attraction of the normal law. Then their common characteristic function $\phi(t)$ is given by (cf. Ibragimov and Linnik [6], p. 91),

$$
\log \phi(t)=-\frac{t^{2}}{2} H\left(|t|^{-1}\right)(1+o(1))
$$

where $H(t)$ is a slowly varying function as $t \rightarrow \infty$ and is given by the formula

$$
H(x)=-\int_{0}^{x} u^{2} d \psi(x)=\int_{-\infty}^{x} u^{2} d G(u),
$$

where $\Psi(x)=1-G(x)+G(x)$ and $G(x)$ is the common distribution function.

Also

$$
|\dot{\phi}(t)| \sim \exp \left\{-\frac{t^{2}}{2} H\left(|t|^{-1}\right)\right\} .
$$

If we put $H(1 / t)=L(t)$, then $L(t)$ is slowly varying as $t \rightarrow 0$. Then (2.3) and (2.5) will take the forms

$$
\log \phi(t)=-\frac{t^{2}}{2} L(|t|)(1+o(1))
$$




$$
|\phi(t)| \sim \exp \left\{-\frac{t^{2}}{2} L(|t|)\right\}
$$

respectively. Since $L(|t|)$ is positive we can write the characteristic function $\phi$ as

$$
\phi(t)=\exp \left\{-\frac{t^{2}}{2} h(t)\right\}
$$

where $h(t)=L(|t|)(1+o(1))$ with the property

$$
h(t)=\operatorname{Re} h(t)(1+o(1)),
$$

as

$$
\operatorname{Re} h(t)=L(|t|)(1+o(1)) .
$$

Now $h(t)$ is slowly varying as $t \rightarrow 0$, since for $\gamma>0$,

$$
\lim _{t \rightarrow 0} \frac{h(\gamma(t))}{h(t)}=\lim _{t \rightarrow 0} \frac{L(\gamma|t|)(1+o(1))}{L(|t|)(1+o(1))}=1 .
$$

Consider the function $h_{1}(t)$ determined by

$$
h_{1}(t)=\left[\begin{array}{l}
\operatorname{Re} h(t) \text { if } V\left(X_{r}\right)=\infty, \\
\sigma^{2} \text { if } V\left(X_{r}\right)=\sigma^{2}<\infty .
\end{array}\right.
$$

Clearly $h_{1}(t)$ is slowly varying in a neighborhood of the origin. By (2.7),

$$
h(t)=h_{1}(t)(1+o(1)) \text {, in both cases as } t \longrightarrow 0 \text {. }
$$

Since expectation is zero, by virtue of (2.4), we have

$$
\lim _{x \rightarrow \infty} H(x)=\int_{-\infty}^{\infty} u^{2} d G(u)=\sigma^{2} .
$$

Therefore when variance is infinite, $\lim _{x \rightarrow \infty} H(x)=\infty$, so that $\lim _{t \rightarrow 0} L(t)=\infty$. Thus we have for infinite variance,

$$
\lim _{t \rightarrow 0} h_{1}(t)=\infty \text {. }
$$

THEOREM 2. Let

$$
f(x)=\sum_{r=0}^{n} a_{r} X_{r} x^{r}
$$

be a polynomial of degree $n$, where $X_{r}$ 's are independent and identically distributed random variables which belong to the domain of attraction of the normal law, have zero means and $P\left\{X_{r} \neq 0\right\}>0$. Let $a_{0}, a_{1}, a_{2} \cdots a_{n}$ be nonzero real number such that $\left(k_{n} / t_{n}\right)=0(\log n)$ 
where $k_{n}=\max _{0 \leqq r \leqq n}\left|a_{r}\right|$ and $t_{n}=\min _{0 \leqq r \leqq n}\left|a_{r}\right|$. Then there exists $a$ positive $n_{0}$ such that the number of real roots of $f(x)=0$ is at least $\mu\left\{\log n / \log \left(\left(k_{n} / t_{n}\right) \log \log n\right)\right\}^{1 / 2}$ outside a set of measure at most $\mu^{\prime}\left\{\log \left(\left(k_{n_{0}} / t_{n_{0}}\right) \log \log n_{0}\right) / \log n_{0}\right\}^{(1-\varepsilon) / 2}$ for $n>n_{0}$ and $1>\varepsilon>0$.

3. Proof of the Theorem 2. Take constants $A$ and $D$ such that

$$
0<D<1 \text { and } A>1
$$

Let

$$
\lambda_{m}=m \log \log n
$$

Let

$$
M_{n}=\left[d^{2}(\log \log n)^{2}\left(k_{n} / t_{n}\right)^{2}(\sqrt{2}+1)^{2}(A e / D)\right]+1,
$$

where $b$ is a positive constant greater than one whose choice will be made later and $[x]$ denotes the greatest integer not exceeding $x$.

It follows from (3.3) that

$$
\mu_{1}\left(\frac{k_{n}}{t_{n}} \log \log n\right)^{2} \leqq M_{n} \leqq \mu_{2}\left(\frac{k_{n}}{t_{n}} \log \log n\right)^{2} .
$$

We define

$$
\phi(x)=x^{[\log x]+x} .
$$

Let $k$ be the integer determined by

$$
\phi(8 k+7) M_{n}^{8 k+7} \leqq n<\phi(8 k+11) M_{n}^{8 k+11} .
$$

The first inequality of (3.5) gives

$$
\log \phi(8 k+7)+(8 k+7) \log M_{n} \leqq \log n,
$$

or

$$
(8 k+7) \log M_{n}<\log n,
$$

which by help of (3.4) yields

$$
k<\frac{\mu \log n}{\log \left(\frac{k_{n}}{t_{n}} \log \log n\right)} .
$$

Again the right hand side inequality of (3.4) gives

$$
\begin{aligned}
\log n & <\log \phi(8 k+11)+(8 k+11) \log M_{n} \\
& =(\log (8 k+11)+8 k+11) \log (8 k+11)+(8 k+11) \log M_{n} \\
& <2(8 k+11)^{2}+(8 k+11) \log M_{n}<\mu_{3} k^{2} \log M_{n},
\end{aligned}
$$


whence by (3.4), we have

$$
\mu_{0}\left(\frac{\log n}{\log \left(k_{n} / t_{n} \log \log n\right)}\right)^{1 / 2}<k .
$$

Therefore

$$
\mu_{0}\left(\frac{\log n}{\log \left(k_{n} / t_{n} \log \log n\right)}\right)^{1 / 2}<k<\mu \frac{\log n}{\log \left(k_{n} / t_{n} \log \log n\right)} .
$$

Since $\left(k_{n} / t_{n}\right)=o(\log n)$ by hypothesis, it follows from (3.7), that $k \rightarrow \infty$ as $n \rightarrow \infty$.

We have $f\left(x_{m}\right)=U_{m}+R_{m}$ at the points

$$
x_{m}=\left(1-\frac{1}{\phi(4 m+1) M_{n}^{4 m}}\right)^{1 / 2}
$$

for $m=[k / 2]+1,[k / 2]+2 \cdots k$, where

$$
U_{m}=\sum_{1} a_{r} X_{r} x_{m}^{r}
$$

and

$$
R_{m}=\left(\sum_{2}+\sum_{3}\right) a_{r} X_{r} x_{m}^{r},
$$

the index $r$ ranging from $\phi(4 m-1) M_{n}^{4 m-1}+1$ to $\phi(4 m+3) M_{n}^{4 m+3}$ in $\sum_{1}$, from 0 to $\phi(4 m-1) M_{n}^{4 m-1}$ in $\sum_{2}$ and from $\phi(4 m+3) M_{n}^{4 m+3}+1$ to $n$ in $\sum_{3}$. (We shall use the notations $\sum_{1}, \Sigma_{2}$ and $\sum_{3}$ to carry the above meaning throughout this paper.)

We have also

$$
f\left(x_{2 m}\right)=U_{2 m}+R_{2 m}, f\left(x_{2 m+1}\right)=U_{2 m+1}+R_{2 m+1} .
$$

By (3.7), we have $2 k+1<n$ for large $n$. Also the maximum index in $U_{2 m+1}$ for $m=k$ is $\phi(8 k+7) M_{n}^{8 k+7}$, which by (3.6) is consistent with (3.9).

We define normalizing constants $V_{m}$ starting from the relation

$$
\left(1 / V_{m}^{2}\right) \sum_{1} a_{r}^{2} x_{m}^{2 r} h_{1}\left(a_{r} x_{m}^{r} \theta / V_{m}\right)
$$

where $\theta$ is a small positive number whose final choice will be dealt with later. Such normalizing constants $V_{m}$ always exist when $\theta$ is sufficiently small. (Cf. Ibragimov and Maslova [7], p. 232.)

Now if $V\left(X_{r}\right)=\infty$, we have

$$
V_{m}^{2}=\sum_{1} a_{r}^{2} x_{m}^{2 r} h_{1}\left(a_{r} x_{m}^{r} \theta / V_{m}\right)>\sum_{1} a_{r}^{2} x_{m}^{2 r}
$$

(by (2.9), since $\theta$ is small), 


$$
\begin{aligned}
& >t_{n}^{2} \sum_{\phi(4 m-1) M_{n}^{4 m-1}+1}^{\phi(4 m+1) M_{n}^{4 m}} x_{m}^{2 r} \\
& >t_{n}^{2}\left\{\phi(4 m+1) M_{n}^{4 m}-\phi(4 m-1) M_{n}^{4 m-1}\right\}\left\{1-\frac{1}{\phi(4 m+1) M_{n}^{4 m}}\right\}^{\phi(4 m+1) M_{n}^{4 m}} \\
& >t_{n}^{2} \phi(4 m+1) M_{n}^{4 m}(D / A e) .
\end{aligned}
$$

Or

$$
M_{n}^{2 m}<(A e / D \phi(4 m+1))^{1 / 2}\left(V_{m} / t_{n}\right) .
$$

Again if $V\left(X_{r}\right)=\sigma^{2}<\infty$, then

$$
\begin{aligned}
V_{m}^{2} & =\sigma^{2} \sum_{1} a_{r}^{2} x_{m}^{2 r} \\
& >\sigma^{2} \phi(4 m+1) M_{n}^{4 m}(D / A e) .
\end{aligned}
$$

Or

$$
M_{n}^{2 m}<(A e / D \phi(4 m+1))^{1 / 2}\left(V_{m} / \sigma t_{n}\right) .
$$

The following lemmas are necessary for the proof of the theorem.

\section{LEMMA 1.}

$$
\left|\sum_{2} a_{r} X_{r} x_{m}^{r}\right|<\lambda_{m} W_{m}
$$

except for a set of measure at most $\mu / \lambda_{m}^{2-\varepsilon}$ for $\varepsilon>0$, where

$$
W_{m}^{2}=\sum_{2} a_{r}^{2} x_{m}^{2 r} h_{1}\left(a_{r} x_{m}^{r} \theta / W_{m}\right) .
$$

Proof. The characteristic function of $\left(1 / W_{m}\right) \sum_{2} a_{r} X_{r} x_{m}^{r}$ is given by

$$
\phi_{m}(t)=\exp \left(-\frac{t^{2}}{2} h_{m}(t)\right)
$$

where

$$
h_{m}(t)=\left(1 / W_{m}^{2}\right) \sum_{2} a_{r}^{2} x_{m}^{2 r} h_{1}\left(a_{r} x_{m}^{r} \theta / W_{m}\right) .
$$

We have by Theorem 1 for $|t|<\theta$,

$$
\begin{array}{r}
h_{1}\left(a_{r} x_{m}^{r} t / W_{m}\right)\left(h_{1}\left(a_{r} x_{m}^{r} \theta / W_{m}\right)\right)^{-1}=\frac{L\left(\left|a_{r} x_{m}^{r} t / W_{m}\right|\right)(1+o(1))}{L\left(\left|a_{r} x_{m}^{r} \theta / W_{m}\right|\right)(1+o(1))} \\
=\frac{c\left(\left|a_{r} x_{m}^{r} t / W_{m}\right|\right)(1+o(1))}{c\left(\left|a_{r} x_{m}^{r} \theta / W_{m}\right|\right)(1+o(1))} \exp \left\{\int_{\mid a_{r} x_{m}^{r} x^{t / W_{m} \mid}}^{\left|a_{r} x^{r} r_{\mid W}\right|} \frac{\bar{\varepsilon}(u)}{u} d u\right\},
\end{array}
$$

where $\lim _{x \rightarrow 0} c(x)=c \neq 0, \lim _{x \rightarrow 0} \bar{\varepsilon}(x)=0$. Again since $\lim _{u \rightarrow 0} \bar{\varepsilon}(u)=0$, 
there exists a positive $t_{0}$ such that for $|t|<\theta<t_{0}^{-1}$ and $\varepsilon>0$, $|\bar{\varepsilon}(u)|<\varepsilon$. Thus we have

$$
h_{1}\left(a_{r} x_{m}^{r} t / W_{m}\right) \leqq\left|\frac{t}{\theta}\right|^{-\varepsilon} h_{1}\left(a_{r} x_{m}^{r} \theta / W_{m}\right)
$$

Now

$$
\begin{aligned}
\operatorname{Re} h_{m}(t) & =\left(1 / W_{m}^{2}\right) \sum_{2} a_{r}^{2} x_{m}^{2 r} h_{1}\left(a_{r} x_{m}^{r} t / W_{m}\right) \\
& \leqq|t / \theta|^{-\varepsilon}\left(1 / W_{m}^{2}\right) \sum_{2} a_{r}^{2} x_{m}^{2 r} h_{1}\left(a_{r} x_{m}^{r} \theta / W_{m}\right) \leqq|t / \theta|^{-\varepsilon} \\
& (\text { by (3.13)) } .
\end{aligned}
$$

But by (2.7), $h_{m}(t)=\operatorname{Re} h_{m}(t)(1+o(1))$ as $t \rightarrow 0$. Therefore for $|t|<t_{0}^{-1}$ and $\varepsilon>0$, we have

$$
\left.\left|h_{m}(t)<\mu_{1}\right| t\right|^{-\varepsilon} .
$$

Thus in a neighborhood of zero,

$$
\left|\phi_{m}(t)-1\right|=\left|\exp \left\{-\frac{t^{2}}{2} h_{m}(t)\right\}-1\right| \leqq \mu_{1}|t|^{2-\varepsilon} .
$$

By Gnedenko and Kolmogorov [5],

$$
\begin{aligned}
& P\left\{\left|\sum_{2} a_{r} X_{r} x_{m}^{r}\right|>\lambda_{m} W_{m}\right\}<2-\left|\left(\lambda_{m} / 2\right) \int_{-2 / \lambda_{m}}^{2 / \lambda_{m}} \phi_{m}(t) d t\right| \\
& \quad \leqq\left(\lambda_{m} / 2\right) \int_{-2 / \lambda_{m}}^{2 / \lambda_{m}}\left|\phi_{m}(t)-1\right| d t \leqq \lambda_{m} \mu_{1} \int_{0}^{2 / \lambda_{m}}|t|^{2-\varepsilon} d t \quad \text { (by (3.15)) }, \\
& \quad \leqq \mu / \lambda_{m}^{2-\varepsilon} .
\end{aligned}
$$

Hence the result.

Adopting the above procedure we can also prove the following lemma.

LEMMA 2.

$$
\left|\sum_{3} a_{r} X_{r} x_{m}^{r}\right|<\lambda_{m} Z_{m}
$$

except for a set of measure at most $\mu / \lambda_{m}^{2-\varepsilon}$ where

$$
Z_{m}^{2}=\sum_{2} a_{r}^{2} x_{m}^{2 r} h_{1}\left(a_{r} x_{m}^{r} \theta / Z_{m}\right)
$$

Now we proceed to estimate $R_{m}$. By virtue of Lemma 1 and Lemma 2, we have

$$
\left|R_{m}\right|<\lambda_{m}\left(W_{m}+Z_{m}\right),
$$

for sufficiently large value of $m$. 
Now if $V\left(X_{r}\right)=\infty$, we have

$$
\left|R_{m}\right|<\lambda_{m} k_{n} d\left\{\left(\sum_{2} x_{m}^{2 r}\right)^{1 / 2}+\left(\sum_{3} x_{m}^{2 r}\right)^{1 / 2}\right\}
$$

where

$$
d=\max _{0 \leqq r \leqq n}\left\{\left(h_{1}\left(a_{r} x_{m}^{r} \theta / W_{m}\right)\right)^{1 / 2},\left(h_{1}\left(a_{r} x_{m}^{r} \theta / Z_{m}\right)\right)^{1 / 2}\right\}
$$

We have

$$
\begin{array}{r}
\frac{\phi(4 m+3)}{\phi(4 m+1)}=\frac{(4 m)^{[\log (4 m+3)]+4 m+3}(1+3 / 4 m)^{[\log (4 m+3)]+4 m+3}}{(4 m)^{[\log (4 m+1)]+4 m+1}(1+1 / 4 m)^{[\log (4 m+1)]+4 m+1}} \\
>(4 m)^{\log (4 m+3 / 4 m+1)+2}=16 m^{2}(4 m)^{\log (4 m+3 / 4 m+1)}>m^{2} .
\end{array}
$$

Therefore

$$
\phi(4 m+3)>m^{2} \phi(4 m+1)
$$

and similarly

$$
\dot{\phi}(4 m+1)>m^{2} \phi(4 m-1) .
$$

Now

$$
\begin{aligned}
\sum_{2} x_{m}^{2 r} & <1+\phi(4 m-1) M_{n}^{4 m-1}<2 \phi(4 m-1) M_{n}^{4 m-1} \\
& <\left(2 / m^{2}\right) \dot{\phi}(4 m+1) M_{n}^{4 m-1}(\text { by }(3.18))
\end{aligned}
$$

and

$$
\begin{gathered}
\left(\sum_{3} x_{m}^{2 r}\right)<\left(\sum_{m^{2} \phi(4 m+1) M_{n}^{4 m+1}} x_{m}^{2 r}\right) \\
\text { (since by } \left.(3.17), m^{2} \phi(4 m+1)<m^{2} \phi(4 m+3)\right), \\
=\phi(4 m+1) M_{n}^{4 m}\left\{M_{n} 1-\frac{1}{\phi(4 m+1) M_{n}^{4 m}}\right\}^{m 2 \phi(4 m+1) M_{n}^{4 m+1}} \\
<\phi(4 m+1) M_{n}^{4 m} e^{-m^{2} M_{n}}<\phi(4 m+1) M_{n}^{4 m}\left(m^{2} M_{n}\right)^{-1}\left(\text { since } e^{-x}<x^{-1}\right), \\
=\left(1 / m^{2}\right) \phi(4 m+1) M_{n}^{4 m-1} .
\end{gathered}
$$

Hence by (3.19) and (3.20) we have from (3.16),

$$
\begin{aligned}
&\left|R_{m}\right|<d \lambda_{m} \frac{(\sqrt{2}+1)}{m}\{\phi(4 m+1)\}^{1 / 2}\left(M_{n}^{2 m} / M_{n}^{1 / 2}\right) \\
&<\frac{d(\sqrt{2+1})(A e / D)^{1 / 2}\left(k_{n} / t_{n}\right) \log \log n V_{m}}{M_{n}^{1 / 2}} \\
& \text { (by (3.2) and (3.11)) } \quad<V_{m}(\text { by }(3.3)) .
\end{aligned}
$$

Again if $V\left(X_{r}\right)=\sigma^{2}<\infty$, then 


$$
\begin{aligned}
\left|R_{m}\right| & \left.<\lambda_{m} \sigma\left\{\sum_{2} x_{m}^{2 r}\right)^{1 / 2}+\left(\sum_{3} x_{m}^{2 r}\right)^{1 / 2}\right\} \\
& <\frac{\log \log n(\sqrt{2}+1)(D / A e)^{1 / 2}\left(k_{n} / t_{n}\right) V_{m}}{M_{n}^{1 / 2}}
\end{aligned}
$$

(by (3.2) and (3.12)),

$$
<\frac{d(\sqrt{2}+1)\left(k_{n} / t_{n}\right) \log \log n V_{m}}{M_{n}^{1 / 2}} . \quad(\text { since } d>1 .)<V_{m} .
$$

Since $k \rightarrow \infty$ as $n \rightarrow \infty$, it follows that when $n$ is sufficiently large

$$
\left|R_{m}\right|<V_{m}
$$

for $m=[k / 2]+1,[k / 2]+2, \cdots, k$, except for a set of measure at most

$$
\left(\mu / \lambda_{m}^{2-\varepsilon}\right) .
$$

Thus we have $\left|R_{2 m}\right|<V_{2 m}$ and $\left|R_{2 m+1}\right|<V_{2 m+1}$ for $m=m_{0}$, $m_{0}+1, \cdots, k$, where $m_{0}=[k / 2]+1$.

The measure of the exceptional set is at most

$$
\left(\mu^{\prime} / \lambda_{2 m}^{2-\varepsilon}\right)+\left(\mu^{\prime} / \lambda_{2 m+1}^{2-\varepsilon}\right)<\left(\mu^{\prime} / \lambda_{m}^{2-\varepsilon}\right) .
$$

Again we proceed to estimate

$$
\begin{aligned}
P^{*}=P\left\{U_{2 m}>\right. & \left.V_{2 m}, U_{2 m+1}<-V_{2 m+1}\right\} \cup\left\{U_{2 m}<-V_{2 m}, U_{2 m+1}>V_{2 m+1}\right\} \\
= & P\left\{U_{2 m}>V_{2 m}\right\} P\left\{U_{2 m+1}<-V_{2 m+1}\right\} \\
& +P\left\{U_{2 m}<-V_{2 m}\right\} P\left\{U_{2 m+1}>V_{2 m+1}\right\} .
\end{aligned}
$$

Let $G_{m}(x)$ and $g_{m}(t)$ be the distribution function and the characteristic function of $\left(U_{m} / V_{m}\right)$ respectively. Then

$$
g_{m}(t)=\exp \left\{\frac{t^{2}}{2} \frac{1}{V_{m}^{2}} \sum_{1} a_{r}^{2} x_{m}^{2 r} h\left(a_{r} x_{m}^{r} t / V_{m}\right)\right\} .
$$

Let

$$
F(x)=\int_{-\infty}^{x} \exp \left(-u^{2} / 2\right) d u .
$$

It follows from (3.11) and (3.12) that $V_{m} \rightarrow \infty$ as $m \rightarrow \infty$ and then $\left(a_{r} x_{m}^{r} t / V_{m}\right) \rightarrow 0$. Therefore when $m \rightarrow \infty$ we have by (2.8),

$$
h\left(a_{r} x_{m}^{r} t / V_{m}\right)=h_{1}\left(a_{r} x_{m}^{r} t / V_{m}\right)(1+o(1))
$$

and by Theorem 1, it can be shown that

$$
h_{1}\left(a_{r} x_{m}^{r} t / V_{m}\right)=\|\theta / t\|^{o(1)} h_{1}\left(a_{r} x_{m}^{r} \theta / V_{m}\right)(1+o(1))
$$

and as such 


$$
\begin{aligned}
g_{m}(t) & =\exp \left\{-\frac{t^{2}}{2} \frac{1}{V_{m}^{2}} \sum_{1} a_{r}^{2} x_{m}^{2 r} h_{1}\left(a_{r} x_{m}^{r} \theta / V_{m}\right)\left|\frac{\theta}{t}\right|^{o(1)}(1+o(1))(1+o(1))\right\} \\
& =\exp \left\{\frac{|t|^{2-o(1)}}{2}\left|\frac{\theta}{t}\right|^{o(1)}(1+o(1))\right\}(\text { by }(3.10)) .
\end{aligned}
$$

Therefore as $m \rightarrow \infty, g_{m}(t) \rightarrow \exp \left(-t^{2} / 2\right)$ uniformly in any bounded interval of $t$-values. Hence

$$
\sup _{x}\left|G_{m}(x)-F(x)\right|=o(1) \text {. }
$$

Then we have for $\varepsilon>0$,

$$
\left|G_{m}(-1)-F(-1)\right|<\varepsilon
$$

and

$$
\left|G_{2 m+1}(-1)-F(-1)\right|<\varepsilon \text {. }
$$

By (3.25) and (3.26), we have

$$
P\left\{U_{2 m}<-V_{2 m}\right\}>F(-1)-\varepsilon
$$

and

$$
P\left\{U_{2 m+1}<-V_{2 m+1}\right\}>F(-1)-\varepsilon .
$$

In the similar way using (3.24) we can show that

$$
P\left\{U_{2 m}>V_{2 m}\right\}>1-F(1)-\varepsilon
$$

and

$$
P\left\{U_{2 m+1}>V_{2 m+1}\right\}>1-F(1)-\varepsilon .
$$

Therefore $P^{*}>2(F(1)-\varepsilon)(1-F(1)-\varepsilon)$. Thus $P^{*}$ is greater than a quantity which tends to $2 F(-1)(1-F(1))$ as $m \rightarrow \infty$ with $n$. This limit being positive we conclude that

$$
P^{*}>\delta>0 \text { for all large } m .
$$

Now we define events $E_{m}$ and $F_{m}$ as follows:

$$
\begin{aligned}
& E_{m}=\left\{U_{2 m}>V_{2 m}, U_{2 m+1}<-V_{2 m+1}\right\}, \\
& F_{m}=\left\{U_{2 m}<-V_{2 m}, U_{2 m+1}>V_{2 m+1}\right\} .
\end{aligned}
$$

By (3.27), we have

$$
P\left\{E_{m} \cup F_{m}\right\}>\delta>0 .
$$

Let $P\left\{E_{m} \cup F_{m}\right\}=\delta_{m}$, so that $\delta_{m}>\delta>0$.

Let $y_{m}$ be the random variable such that it takes value 1 on $E_{m} \cup F_{m}$ and 0 elsewhere. In otherwords, 


$$
y_{m}=\left[\begin{array}{l}
1 \text { with probability } \delta_{m}, \\
0 \text { with probability } 1-\delta_{m} .
\end{array}\right.
$$

The $y_{m}$ 's are thus independent random variables with $E\left(y_{m}\right)=0$ and $V\left(y_{m}\right)=\delta_{m}-\delta_{m}^{2}<1$. We write

$$
\boldsymbol{z}_{m}=\left[\begin{array}{ll}
0 & \text { if }\left|R_{2 m}\right|<V_{2 m} \text { and }\left|R_{2 m+1}\right|<V_{2 m+1} \\
1 & \text { otherwise }
\end{array}\right.
$$

Moreover, we have $f\left(x_{2 m}\right)=U_{2 m}+R_{2 m}$ and $f\left(x_{2 m+1}\right)=U_{2 m+1}+R_{2 m+1}$. Let $\alpha_{m}=y_{m}-y_{m} z_{m}$. Now $\alpha_{m}=1$ only if $y_{m}=1$ and $z_{m}=0$, which implies the occurrence of one of the events

$$
\begin{aligned}
& U_{2 m}>V_{2 m},\left|R_{2 m}\right|<V_{2 m} ; \\
& U_{2 m+1}<-V_{2 m+1},\left|R_{2 m+1}\right|<V_{2 m+1}, \\
& U_{2 m}<-V_{2 m},\left|R_{2 m}\right|<V_{2 m} ; \\
& U_{2 m+1}>V_{2 m+1},\left|R_{2 m+1}\right|<V_{2 m+1} .
\end{aligned}
$$

It is obvious that (i) implies $f\left(x_{2 m}\right)>0$ and $f\left(x_{2 m+1}\right)<0$, and (ii) implies that $f\left(x_{7 m}\right)<0$ and $f\left(x_{2 m+1}\right)>0$. Thus if $\alpha_{m}=1$, there is a root of the polynomial in the interval $\left(x_{2 m}, x_{2 m+1}\right)$. Hence the number of roots in $\left(x_{2 m_{0}}, x_{2 k+1}\right)$ must exceed $\sum_{m=m_{0}}^{k} \alpha_{m}$.

We appeal to the strong law of large numbers in the following form. The technique has been earlier used by Evans [4], Samal and Mishra [12] and [13].

Let $y_{1}, y_{2}, \cdots$, be a sequence of independent random variables identically distributed with $V\left(y_{i}\right)<1$ for all $i$, then for each $\varepsilon>0$,

$$
P\left\{\sup _{k \geqq k_{0}}\left|\frac{1}{k} \sum_{i=1}^{k}\left(y_{i}-E\left(y_{i}\right)\right)\right|>\varepsilon\right\}<B / \varepsilon^{2} k_{0},
$$

where $B$ is a positive constant.

In the present case,

$$
\begin{gathered}
\left|\sum_{m=m_{0}}^{k}\left(\alpha_{m}-E\left(y_{m}\right)\right)\right| \leqq\left|\sum_{m=m_{0}}^{k}\left(y_{m}-E\left(y_{m}\right)\right)\right|+\left|\sum_{m=m_{0}}^{k} y_{m} z_{m}\right| \\
\left.\leqq\left|\sum_{m=m_{0}}^{k}\left(y_{m}-E\left(y_{m}\right)\right)\right|+\left|\sum_{m=m_{0}}^{k} z_{m}\right| \text { (since } y_{m} \leqq 1\right) .
\end{gathered}
$$

Since $E\left(z_{m}\right)=1 \cdot P\left\{z_{m}=1\right\}<P\left\{\left|R_{m}\right|>V_{m}\right\}$ we have from (3.21),

$$
E\left(z_{m}\right)<\mu / \lambda_{m}^{2-\varepsilon} .
$$

Now we have

$$
P\left\{\sum_{m=m_{0}}^{k} z_{m} \geqq\left(k-m_{0}+1\right) \varepsilon_{1}\right\}<\mu / \lambda_{m_{0}}^{2-\varepsilon} .
$$


Hence outside an exceptional set of measure at most

we have

$$
\sum_{\left(k-m_{0}+1\right) \geqq k_{0}}\left(\mu / \lambda_{m_{0}}^{2-\varepsilon}\right),
$$

and therefore,

$$
\sup _{\left(k-m_{0}+1\right) \geqq k_{0}}\left(1 /\left(k-m_{0}+1\right)\right) \sum_{m=m_{0}}^{k} z_{m}<\varepsilon_{1}
$$

$$
\begin{aligned}
& \sup _{\left(k-m_{0}+1\right) k \geqq k_{0}}\left(1 /\left(k-m_{0}+1\right)\right)\left|\sum_{m=m_{0}}^{k}\left(\alpha_{m}-E\left(y_{m}\right)\right)\right| \\
& \quad \leqq \sup _{\left(k-m_{0}+1\right) \geqq k_{0}}\left(1 /\left(k-m_{0}+1\right)\right)\left|\sum_{m=m_{0}}^{k}\left(y_{m}-E\left(y_{m}\right)\right)\right|+\varepsilon_{1} .
\end{aligned}
$$

Now by using strong law of large numbers,

$$
\begin{gathered}
P\left\{\sup _{\left(k-m_{0}+1\right) \geqq k_{0}}\left|\left(1 /\left(k-m_{0}+1\right)\right) \sum_{m=m_{0}}^{k}\left(\alpha_{m}-E\left(y_{m}\right)\right)\right|>\varepsilon\right\} \\
<B /\left(\varepsilon-\varepsilon_{1}\right)^{2} k_{0}=\mu / k_{0} .
\end{gathered}
$$

By (3.1)

$$
\lambda_{m_{0}}^{2-\varepsilon}=\left(m_{0} \log \log n\right)^{2-\varepsilon} .
$$

For large $n, m_{0} \log \log n>m_{0}$, and therefore

$$
\sum\left(\mu / \lambda_{m_{0}}^{2-\varepsilon}\right)<\sum\left(\mu / m_{0}^{2-\varepsilon}\right) .
$$

Hence outside a set $S_{k_{0}}$, where

$$
P\left(S_{k_{0}}\right)<\mu / k_{0}+\sum_{\left(k-m_{0}+1\right) \geqq k_{0}}\left(\mu / m_{0}^{2-\varepsilon}\right),
$$

we have

$$
\left(1 /\left(k-m_{0}+1\right)\right)\left|\sum_{m=m_{0}}^{k}\left(\alpha_{m}-E\left(y_{m}\right)\right)\right|<\varepsilon .
$$

Also

Therefore,

$$
E\left(y_{m}\right)=\delta_{m}>\delta
$$

$$
\begin{gathered}
N_{n}>\sum_{m=m_{0}}^{k} \alpha_{m}>\sum_{m=m_{0}}^{k} \delta-\left(k-m_{0}+1\right) \varepsilon>(k-[k / 2]) \\
>\mu\left(\log n / \log \left(\left(k_{n} / t_{n}\right) \log \log n\right)\right)^{1 / 2}(\mathrm{by}(3.7)),
\end{gathered}
$$

for all $k$ such that $k-m_{0}+1>k_{0}$, or in otherwords for all $n>n_{0}$. Now

$$
\begin{aligned}
P\left(S_{k_{0}}\right) & <\left(\mu / k_{0}\right)+\mu \sum_{k \geq\left(2 k_{0}-1\right)}\left(1 / m_{0}\right)^{2-\varepsilon} \\
& =\frac{\mu}{k_{0}}+\mu\left\{\frac{1}{k_{0}^{2-\varepsilon}}+2\left(\frac{1}{k_{0}^{2-\varepsilon}+1}+\frac{1}{k_{0}^{2-\varepsilon}+2}+\cdots\right)\right\} \\
& <\left(\mu / k_{0}\right)+2 \mu \sum_{k \geq k_{0}}\left(1 / k^{2-\varepsilon}\right) .
\end{aligned}
$$


It can be easily shown that for $0<\varepsilon<1$,

$$
\sum_{k \geq k_{0}}\left(1 / k^{2-\varepsilon}\right)<\left(1 /(1-\varepsilon) k_{0}^{1-\varepsilon}\right)
$$

Hence

$$
P\left(S_{k_{0}}\right)<\left(\mu / k_{0}\right)+\left(1 /(1-\varepsilon) k_{0}^{1-\varepsilon}\right)<\mu_{1} / k_{0}^{1-\varepsilon}
$$

(since by hypothesis $0<\varepsilon<1, k_{0}>k_{0}^{(1-\varepsilon) / 2}$ ),

$$
<\mu^{\prime}\left\{\log \left(\left(k_{n_{0}} / t_{n_{0}}\right) \log \log n_{0}\right) / \log n_{0}\right\}^{1-\varepsilon}(\text { by }(3.7)) \text {. }
$$

The authors are thankful to the referee for his valuable comments.

\section{REFERENCES}

1. J. E. A. Dunnage, The number of real zeros of a class of random algebraic polynomials, Proc. London Math. Soc., (3) 18 (1968), 439-460.

2. - The number of real zeros of a class of random algebraic polynomials II, Quart. J. Math. Oxford, (2), 21 (1970), 309-319.

3. - The number of real zeros of a class of random algebraic polynomials III, J. London Math. Soc., (2) 6 (1972), 143-152.

4. E. A. Evans, On the number of real roots of a random algebraic equation, Proc. London Math. Soc., 15 (1965), 731-749.

5. B. V. Gnedenko and A. N. Kolmogorov, Limit Distribution of Sums of Independent Random Variables, Addison Wesley, Inc., 1954.

6. I. A. Ibragimov and Yu. V. Linnik, Independent and Stationary Sequence of Random Variables, Wolters-Noordhoff Publishing Groningen, 1972.

7. I. A. Ibragimov and N. B. Maslova, On the expected number of real zeros of random algebraic polynomial 1. Coefficients with zero means, (Translated by B. Seckler), Theor. Probability Application, XVI, 2 (1971), 228-248.

8. J. E. Littlehood and A. C. Offord, On the number of real roots of a random algebraic equation II, Proc. Cambridge Phil. Soc., 35 (1939), 133-148.

9. M. N. Mishra and N. N. Nayak, On the lower bound of the number of real roots of a random algebraic equation, accepted in the Journal of I. M. S. for publication.

10. G. Samal, On the number of real roots of a random algebraic equation, Proc. Cambridge Phils. Soc., 58 (Part 3) (1962), 433-442.

11. G. Samal and M. N. Mishra, On the lower bound of the number of real roots of a random algebraic equation with infinite variance, Proc. Amer. Math. Soc., 33 (1972), 523-528.

12. On the lower bound of the number of real roots of a random algebraic equation with infinite variance II, Proc. Amer. Math. Soc., 36 (1972), 557-563.

13. On the lower bound of the number of real roots of a random algebraic equation with infinite variance III, Proc. Amer. Math. Soc., 39 (1973). 184-189,

Received August 19, 1980 and in revised form September 16, 1981.

SAMBALPUR UNIVERSITY,

JYOTIVIHAR, BURLA

PIN-768017

Sambalpur, Orissa, India. 


\title{
PACIFIC JOURNAL OF MATHEMATICS
}

\section{EDITORS}

\author{
DONALD BABBITT (Managing Editor) \\ University of California \\ Los Angeles, CA 90024 \\ Hugo Rossi \\ University of Utah \\ Salt Lake City, UT 84112 \\ C. C. MOORE and ARthur Agus \\ University of California \\ Berkeley, CA 94720
}

J. DUGUNDJI

Department of Mathematics

University of Southern California

Los Angeles, CA 90007

R. FINN and J. MILGRAM

Stanford University

Stanford, CA 94305

\section{ASSOCIATE EDITORS}
R. ARENS
E. F. BeCKENBACH
B. H. NeumanN
F. WoLF
K. YoSHIDA

\section{SUPPORTING INSTITUTIONS}

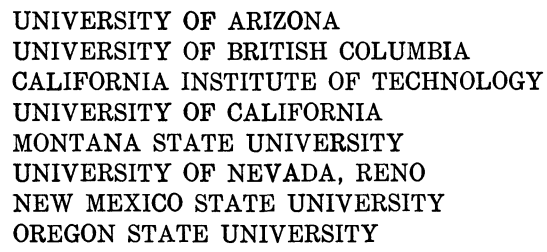

UNIVERSITY OF ARIZONA

UNIVERSITY OF BRITISH COLUMBIA

CALIFORNIA INSTITUTE OF TECHNOLOGY

UNIVERSITY OF CALIFORNIA

MONTANA STATE UNIVERSITY

UNIVERSITY OF NEVADA, RENO

NEW MEXICO STATE UNIVERSITY

OREGON STATE UNIVERSITY

\author{
UNIVERSITY OF OREGON \\ UNIVERSITY OF SOUTHERN CALIFORNIA \\ STANFORD UNIVERSITY \\ UNIVERSITY OF AAWAII \\ UNIVERSITY OF TOKYO \\ UNIVERSITY OF UTAH \\ WASHINGTON STATE UNIVERSITY \\ UNIVERSITY OF WASHINGTON
}

The Supporting Institutions listed above contribute to the cost of publication of this Journal, but they are not owners or publishers and have no responsibility for its content or policies,

Mathematical parers intended for publication in the Pacific Journal of Mathematics should be in typed form or offset-reproduced, (not dittoed), double spaced with large margins. Please do not use built up fractions in the text of the manuscript. However, you may use them in the displayed equations. Underline Greek letters in red, German in green, and script in blue. The first paragraph or two must be capable of being used separately as a synopsis of the entire paper. Please propose a heading for the odd unmbered pages of less than 35 characters. Manuscripts, in triplicate, may be sent to any one of the editors. Please classify according to the scheme of Math. Reviews, Index to Vol. 39. Supply name and address of author to whom proofs should be sent. All other communications should be addressed to the managing editor, or Elaine Barth, University of California, Los Angeles, California, 90024.

50 reprints to each author are provided free for each article, only if page charges have been substantially paid. Additional copies may be obtained at cost in multiples of 50 .

The Pacific Journal of Mathematics is issued monthly as of January 1966, Regular subscription rate: $\$ 114.00$ a year (6 Vol., 12 issues). Special rate: $\$ 57.00$ a year to individual members of supporting institution.

Subscriptions, orders for numbers issued in the last three calendar years, and changes of address shoud be sent to Pacific Journal of Mathematics, P.O. Box 969, Carmel Valley, CA 93924, U.S.A. Old back numbers obtainable from Kraus Periodicals Co., Route 100, Millwood, NY 10546.

PUBLISHED BY PACIFIC JOURNAL OF MATHEMATICS, A NON-PROFIT CORPORATION

Printed at Kokusai Bunken Insatsusha (International Academic Printing Co., Ltd.). 8-8, 3-chome, Takadanobaba, Shinjuku-ku, Tokyo 160, Japan. 


\section{Pacific Journal of Mathematics}

\section{Vol. 103, No. $2 \quad$ April, 1982}

Alberto Alesina and Leonede De Michele, A dichotomy for a class of positive

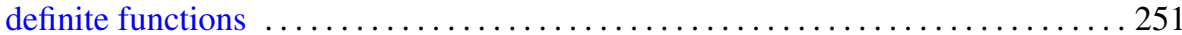

Kahtan Alzubaidy, Rank 2 -groups, $p>3$, and Chern classes . . . . . . . . . . 259

James Arney and Edward A. Bender, Random mappings with constraints on

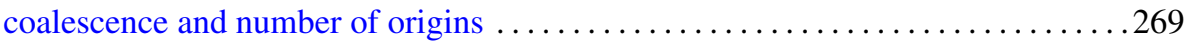

Bruce C. Berndt, An arithmetic Poisson formula . . . . . . . . . . . . . . . 295

Julius Rubin Blum and J. I. Reich, Pointwise ergodic theorems in 1.c.a. groups . . . 301

Jonathan Borwein, A note on $\varepsilon$-subgradients and maximal monotonicity . . . . . . . 307

Andrew Michael Brunner, Edward James Mayland, Jr. and Jonathan Simon,

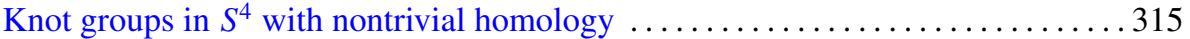

Luis A. Caffarelli, Avner Friedman and Alessandro Torelli, The two-obstacle

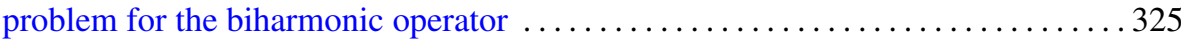

Aleksander Całka, On local isometries of finitely compact metric spaces . . . . . . 337

William S. Cohn, Carleson measures for functions orthogonal to invariant

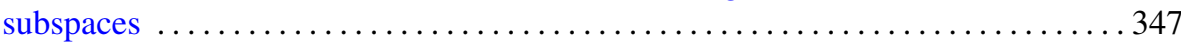

Roger Fenn and Denis Karmen Sjerve, Duality and cohomology for one-relator

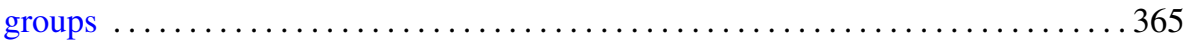

Gen Hua Shi, On the least number of fixed points for infinite complexes . . . . . . . 377

George Golightly, Shadow and inverse-shadow inner products for a class of linear

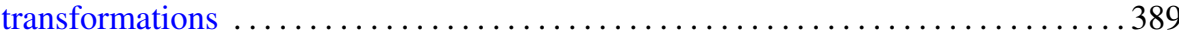

Joachim Georg Hartung, An extension of Sion's minimax theorem with an

application to a method for constrained games $\ldots \ldots \ldots \ldots \ldots \ldots \ldots \ldots \ldots \ldots . \ldots 4$

Vikram Jha and Michael Joseph Kallaher, On the Lorimer-Rahilly and

Johnson-Walker translation planes

Kenneth Richard Johnson, Unitary analogs of generalized Ramanujan sums .

Peter Dexter Johnson, Jr. and R. N. Mohapatra, Best possible results in a class of inequalities

Dieter Jungnickel and Sharad S. Sane, On extensions of nets

Johan Henricus Bernardus Kemperman and Morris Skibinsky, On the

characterization of an interesting property of the arcsin distribution ...

Karl Andrew Kosler, On hereditary rings and Noetherian $V$-rings

William A. Lampe, Congruence lattices of algebras of fixed similarity type. II . . . . 475

M. N. Mishra, N. N. Nayak and Swadeenananda Pattanayak, Strong result for real zeros of random polynomials

Sidney Allen Morris and Peter Robert Nickolas, Locally invariant topologies on free groups

Richard Cole Penney, A Fourier transform theorem on nilmanifolds and nil-theta functions

Andrei Shkalikov, Estimates of meromorphic functions and summability theorems

László Székelyhidi, Note on exponential polynomials

William Thomas Watkins, Homeomorphic classification of certain inverse limit spaces with open bonding maps $\ldots \ldots \ldots \ldots \ldots \ldots \ldots \ldots$

David G. Wright, Countable decompositions of $E^{n}$

Takayuki Kawada, Correction to: "Sample functions of Pólya processes" .

Z. A. Chanturia, Errata: "On the absolute convergence of Fourier series of the 